\title{
COMPLEX MODAL DECOMPOSITION APPLIED TO NEMATODE POSTURING
}

\author{
B. F. Feeny \\ Department of Mechanical Engineering \\ Michigan State University \\ East Lansing, MI 48824 \\ feeny@egr.msu.edu
}

\author{
P. W. Sternberg \\ Division of Biology \\ California Institute of Technology \\ Pasadena, CA 91125 \\ pws@caltech.edu
}

\author{
C. J. Cronin \\ Division of Biology \\ California Institute of Technology \\ Pasadena, CA 91125 \\ cjc@caltech.edu
}

\begin{abstract}
The complex orthogonal decomposition (COD), a process of extracting complex modes from complex ensemble data, is summarized, as is the use of complex modal coordinates. A brief assessment is made on how small levels of noise affect the decomposition. The decomposition is applied to the posturing of a wild Caenorhabditis elegans nematode. The decomposition indicates that the worm has a multi-modal posturing behavior, involving at least a dominant locomotion mode and a secondary, steering mode. The locomotion mode is closer to a pure traveling waveform than the steering mode. The characteristic wavelength of the primary mode was estimated in the complex plane. Frequency was obtained from the complex modal coordinate's complex whirl rate of the complex modal coordinate, and from its fast Fourier transform.
\end{abstract}

\section{Introduction}

In this work, we demonstrate the application of a method of decomposing complex modes to undulatory bio-locomotion. In particular, the method is applied to sensed ensembles of the posturing movements of a nematode as it moves through its medium. Nematodes can be thought of as free-living structures with coordinated excitation from a neuromuscular source. They typically exhibit wave like posturing movements. A complex modal description is appropriate since complex modes can be used to describe oscillations involving traveling waves. The method applied is a complex orthogonal decomposition (COD) [1], which is a generalization of the well known proper orthogonal decom- position (POD).

\subsection{Background on the Decomposition Strategy}

POD, similar to singular value decomposition (SVD), is a tool for extracting modes that optimize the signal energy distribution in a set of measured time series. It has been used to characterize spatial coherence in turbulence and structures [24], the dimension of the dynamics [3-5], empirical modes for reduced order models $[6,7]$, and in system identification $[8,9]$. POD, SVD, and similar tools have been compared for structural applications [10]. In specific circumstances, the POD produces the normal modes of a structure [11-14].

POD is particularly useful if extracting standing wave components, but is less suited for decomposing nonstanding wave components. The COD fills this void. In COD, a complex correlation matrix is formed, and its eigensolution is the basis of the decomposition. A potential biological application area is undulatory bio-locomotion. As such, we apply the tool to nematode posturing movements.

\subsection{Background on Nematodes}

Nematodes are roundworms, nonsegmented worms with digestive, circulatory, and neuromuscular systems. In 1998, Caenorhabditis elegans, a tiny (about $1 \mathrm{~mm}$ in length) nematode which lives freely in the soil, became the first animal whose entire genome was sequenced (The C. elegans Genome Sequencing Consortium, 1998). The genome consists of about $100 \mathrm{mil}-$ lion DNA base pairs, and includes about 20,000 genes. As 
such, C. elegans is a very important model for animal biology and genetics in general. Studies of nematodes can have important implications for all animals, including humans. Since it has a short generation time (three days) and a short life span (two to three weeks), C. elegans is a valuable subject for studying development, neurobiology, and aging [15] (also http://www.wormbook.org).

Nematode locomotion has been useful as an assay for identifying genes and pathways underlying synaptic transmission and its regulation [16-20], and more recently to understand how locomotion is regulated, for example, by mechanosensors [21] and epidermal growth factor [22]. Also, and perhaps most importantly, analysis of locomotion data is crucial for modeling the biomechanics and neural circuits controlling this process [23, 24]. Automated systems now allow the posture of these worms to be extracted from video images [25], but methods for the analysis of changes in posture have not been optimized.

\section{Complex Mode Decomposition}

\subsection{Complex Modes and Wave Motions}

The relationship between complex modes and standing and traveling waves is fundamental to the complex orthogonal decomposition and its interpretation for structural wave motion analysis. Indeed, waves are complex modes. Generally, complex modal motions occur in vibration systems with gyroscopic terms, general damping [28, 29], and asymmetric stiffness matrices (flutter and friction).

The harmonic motion in a complex mode $\mathbf{z}(t)=e^{\alpha t} \mathbf{u}$, where $\mathbf{z}$ is a vector of particle positions, $t$ is time, $\alpha=\gamma+\omega i$, and $\mathbf{u}=$ $\mathbf{c}+\mathbf{d} i$ is a complex mode, with $\gamma, \omega, \mathbf{c}$ and $\mathbf{d}$ being real scalars and vectors, can be expressed in real form as

$$
\mathbf{x}(t)=e^{\gamma t}[\cos (\omega t) \mathbf{c}-\sin (\omega t) \mathbf{d}] .
$$

Thus a complex mode induces an oscillation with a continual transition from the shape $\mathbf{c}$ to the shape $\mathbf{d}$. The relative sizes and degree of independence of $\mathbf{c}$ and $\mathbf{d}$ dictate the "amounts" of standing wave and traveling wave components.

Conversely, e.g. a sinusoidal wave motion of a continuum $y(x, t)=\sin \lambda(x-c t)=\sin \lambda x \cos \omega t-\cos \lambda x \sin \omega t$, where $\omega=$ $c \lambda$. And so

$$
y(x, t)=\operatorname{Re}\left[e^{i \omega t} \sin \lambda x+i e^{i \omega t} \cos \lambda x\right]=\operatorname{Re}(z(x, t)),
$$

where the complex wave motion $z(x, t)=e^{i \omega t}[\sin \lambda x+i \cos \lambda x]$ perfectly matches the form of a continuous complex modal motion. When spatially discretized, the complex wave motion would be $\mathbf{z}=e^{i \omega t}[\mathbf{c}+i \mathrm{~d}]$ where $\mathbf{c}$ is the spatially sampled $\sin \lambda x$ and $\mathbf{d}$ is the spatially sampled $\cos \lambda x$. Then $\mathbf{x}=(\mathbf{z}+\overline{\mathbf{z}}) / 2$ produces a real signal with the above interpretation.
Next, for the complex mode decomposition, we first summarize how to express a real oscillatory signal as a complex analytic signal, for the decomposition process. We then present the decomposition method via an example.

\subsection{Complex Orthogonal Decomposition}

Since the decomposition method is to be applied to complex representations of signals, real measured signals are first extended to the complex world [27]. One way to obtain a complex analytic signal $z(t)$ uniquely from a real signal $y(t)$ by taking at the Fourier transform $\mathcal{F}(y(t))=\tilde{Y}(\omega)$, and truncating the $-i \omega_{n} t$ from $2 \tilde{Y}(\omega)$ to produce $\tilde{Z}(\omega)$. The inverse Fourier transform produces $z(t)=\mathscr{F}^{-1}(\tilde{Z}(\omega))$ [27]. Alternatively, if $y=\operatorname{Re}(z)$, then the $\operatorname{Im}(z)$ is the Hilbert transform of $y$ (e.g. [27]). In practice, either calculation would involve the fast Fourier transform (FFT).

With signals in complex form, $z_{j}, j=1, \ldots, M$, where $M$ is the number of sensors distributed on a structure, we generate vectors $\mathbf{z}_{j}=\left[z_{j}\left(t_{1}\right) \cdots z_{j}\left(t_{N}\right)\right]^{T}$, by sampling at times $t_{1}$ through $t_{N}$. We build an $M \times N$ complex ensemble matrix $Z=\left[\mathbf{z}_{1} \cdots \mathbf{z}_{M}\right]^{T}$.

We construct a complex correlation matrix $\mathbf{R}=\frac{1}{N} \mathbf{Z} \overline{\mathbf{Z}}^{T}$, where the bar indicates complex conjugation. Since $\mathbf{R}$ is complex and Hermitian $\left(\mathbf{R}=\overline{\mathbf{R}}^{T}\right)$, it has real eigenvalues and complex eigenvectors, and its eigenvectors $\mathbf{u}_{i}$ are unitary (complex orthogonal) and satisfy

$$
\overline{\mathbf{u}}_{i}^{T} \mathbf{u}_{j}=0, \quad i \neq j
$$

The eigenvectors $\mathbf{u}_{j}$ of $\mathbf{R}$ are the complex orthogonal modes (COMs) and the eigenvectors $\Lambda_{j}$ are the complex orthogonal values (COVs). We refer to the construction of $\mathbf{R}$ and its eigenvalue problem as a complex orthogonal decomposition (COD for brevity).

For interpretation, suppose we have a signal with $k$ orthogonal modal functions, spatially discretized at $x_{1}, \ldots, x_{M}$, such that $\mathbf{z}(t)=\sum_{j=1}^{k} q_{j}(t) \Phi_{j}$, is an $M$ vector with $m$ components to be extracted, with harmonic modal coordinates $q_{j}(t)=\left(a_{j}+\right.$ $\left.b_{j} i\right) e^{i \omega_{j} t}=f_{j} e^{i \omega_{j t} t}$. (For instance, if the modes are harmonic functions of $x$, as $\phi_{j}(x)=\cos j \lambda x-i \sin j \lambda x$, the samples at $x_{1}, \ldots, x_{M}$ produce $\phi_{j}$.) Then, in matrix form, $\mathbf{z}=\Phi \mathbf{q}_{m}$, where $\Phi$ is an $M \times M$ modal matrix, and $\mathrm{q}_{m}$ is the modal coordinate vector. The signal is uniformly sampled at times $t=t_{1}, \ldots, t_{N}$. As such, the ensemble has the form $\mathbf{Z}_{M \times N}=\Phi \mathbf{Q}_{m}$, where $\mathbf{Q}_{m}$ is an $M \times N$ ensemble matrix of sampled modal coordinates.

The complex correlation matrix is then

$$
\mathbf{R}=\frac{1}{N} \mathbf{Z} \overline{\mathbf{Z}}^{T}=\frac{1}{N} \Phi \mathbf{Q}_{m} \overline{\mathbf{Q}}_{m}^{T} \bar{\Phi}^{T}=\Phi \mathbf{R}_{Q} \bar{\Phi}^{T}
$$

Copyright @ 2009 by ASME 
where $\mathbf{R}_{Q}=\frac{1}{N} \mathbf{Q}_{m} \overline{\mathbf{Q}}_{m}^{T}$. The elements of $\mathbf{R}_{Q}$ are

$$
\begin{aligned}
r_{Q j k} & =\frac{1}{N} \sum_{l=1}^{N} q_{j}\left(t_{l}\right) \bar{q}_{k}\left(t_{l}\right) \\
& =\frac{1}{N} \sum_{l=1}^{N} f_{j} e^{i \omega_{j} t_{l}} \bar{f}_{k} e^{-i \omega_{k} t_{l}}=\frac{f_{j} \bar{f}_{k}}{N} \sum_{l=1}^{N} e^{i\left(\omega_{j}-\omega_{k}\right) t_{l}} .
\end{aligned}
$$

If $\omega_{j} \neq \omega_{k}$, and if $\omega_{j}-\omega_{k} \neq 2 \pi n / \Delta t$ for some integer $n$, then $r_{Q j k} \rightarrow 0$ as $N \rightarrow \infty$. (For discrete-frequency processes, the latter condition is likely, and guaranteed if the sampling frequency exceeds the minimum frequency difference, which could be determined by FFT.)

Thus, if the frequencies are so distinct, $\mathbf{R}_{Q} \rightarrow \mathbf{D}$ as $N$ gets large, where $\mathbf{D}$ is a diagonal matrix of values $d_{j}=$ $\frac{1}{N} \sum_{l=1}^{N} q_{j}\left(t_{l}\right) \bar{q}_{j}\left(t_{l}\right)$. Hence, the matrix $\mathbf{R}_{Q}$ approaches a diagonal of mean squared amplitudes of the complex wave modal coordinates, as $N$ gets large.

In such case, $\phi_{j}$ are (approximate) eigenvectors of $\mathbf{R}$. To see this, consider

$$
\mathbf{R}_{j} \approx \Phi \mathbf{D} \check{\Phi}^{T} \underline{\phi}_{j}=\boldsymbol{\Phi} \mathbf{D} \mathbf{h}_{j}
$$

where $\mathbf{h}_{j}$ is (approximately) a vector of zeros except for the $j$-th element which is one, due to the orthogonality of the complex harmonic basis functions $\phi$. If $\underline{\phi}_{j}$ is a discretization of a onedimensional normalized function, then

$$
1=\int_{0}^{L} \bar{\phi}_{j}(x) \phi_{j}(x) d x \approx \sum_{i=1}^{M}\left|\phi_{j i}\right|^{2} \Delta x_{i}=\Delta x \underline{\phi}_{j}^{T} \underline{\phi}_{j}=\frac{L}{M} \bar{\phi}_{j}^{T} \underline{\phi}_{j}
$$

if $\Delta x_{i}$ are all the same, where $\phi_{j i}$ is the $i$ th element of $\phi_{j}$, and $L$ is the length of the discretized medium. Thus the approximate nonzero value in $\mathbf{h}$ will be $M / L$, as the term $\underline{\phi}_{j}^{T} \underline{\phi}_{j}$ is a rectangular rule approximation of a continuous orthogonality integral in (5).

Then $\Phi D h_{j}=\Phi \underline{\delta}_{j}$, where where $\underline{\delta}_{j}$ is a vector of zeros except for the $j$-th element which is $d_{j} M / L$, and from equation (4),

$$
\mathbf{R}_{\underline{\phi}_{j}} \approx \frac{M}{L} d_{j} \phi_{j}
$$

Then $\phi_{j}$, the discretized modal function, is an approximate eigenvector, proportional to the normalized eigenvector $\mathbf{u}_{j}$, and the associated eigenvalue of the complex correlation matrix $\mathbf{R}$ is $\Lambda_{j} \approx d_{j} M / L$. From equation (3), the mean squared amplitude of the normalized eigenvector is $1 / M$, and from equation (5), when $\mathbf{u}_{j}$ is approximating $\Phi_{j}$, we have $\mathbf{u}_{j} \approx \Phi_{j} \sqrt{L / M}$. Thus the mean squared displacement associated with the $j$-th mode, $q_{j}(t) \underline{\phi}_{j}$, is $d_{j} M / L$ The quality of the approximation depends on the sample resolution, i.e. $M$ and $N$.

Thus, in the example of orthogonal waveforms with harmonic modulations, the complex orthogonal decomposition extracts the complex harmonic waveforms as the COMs and the mean squared modulations as the COVs through the eigenvalue problem associated with the complex correlation matrix $\mathbf{R}$.

Indeed, as waves are a complex generalization of synchronous motions, this COD is a generalization of POD, and will be able to extract both standing and traveling waves, as interpreted from the extracted complex modes.

For POD to recover information about traveling and standing waves requires additional processing of the proper orthogonal modal coordinates (or equivalently, the modal histories that result from SVD or BOD), and recognizing whether two coordinates have the same frequencies and are 90 degrees out of phase. However, the COD will pair these components together, automatically, as real and imaginary parts of a single complex vector. POD can yield equivalent traveling wave components only when the wave components are orthogonal, and only a limited number of wave modes can be captured.

\subsection{Modal Coordinates and Wave Speed}

Similar to $\mathbf{Z}=\Phi \mathbf{Q}_{m}$, we can define $\mathbf{Z}=V \mathbf{V}$, where $\mathbf{V}$ is the matrix of COMs, and $\mathbf{Q}$ is the ensemble of complex orthogonal modal coordinates. If the modes in $\mathbf{V}$ are normalized, then by complex orthogonality (3),

$$
\mathbf{Q}=\overline{\mathbf{V}}^{T} \mathbf{Z}
$$

This is a complex modal coordinate ensemble matrix, the rows of which are the samples of each modal coordinate, $q_{j}(t)$, sampled at $t=t_{1}, \ldots, t_{N}$. Since the vectors making up $\mathbf{V}$ are normalized to unit magnitude, they are unitless, and the units of $\mathbf{Q}$ are the same as those of $\mathbf{Z}$.

The real and imaginary parts of $\mathbf{Q}$ provide the two timemodulation components of the complex wave, representing the time modulations of the $90^{\circ}$-phased components of the wave. Hence, from the modal coordinates in ensemble $\mathbf{Q}$, frequency information can be obtained (by FFT or complex whirl rate) for the wave components. Likewise, the wave number $\lambda_{j}(2 \pi$ over the wavelength) can be obtained (by FFT or the whirl of the complex modal vector) from each of the complex modes $\mathbf{v}_{j}$ from the COD. The wave speed of each wave component is then available, as $c_{j}=\omega_{j} / \lambda_{j}$.

\subsection{Effects of Noise}

Noise can be in issue in both biological and structural systems. Suppose the complex signal has low-level noise in addi- 
tion to pure modal content, such that $\mathbf{Z}=\boldsymbol{\Phi} \mathbf{Q}+\mathbf{\varepsilon E}$, where $\boldsymbol{\varepsilon}$ is "small", and $\mathbf{E E}$ is the ensemble of added noise, which can in turn be written via a modal expansion as $\mathbf{E}=\Phi \mathbf{Q}_{e}$, with normalized orthogonal matrix $\Phi$. Then the complex correlation matrix has the form

$$
\begin{aligned}
N \mathbf{R} & =\mathbf{Z} \overline{\mathbf{Z}}^{T}=(\boldsymbol{\Phi} \mathbf{Q}+\mathbf{\varepsilon} \mathbf{E})\left(\overline{\mathbf{Q}}^{T} \bar{\Phi}^{T}+\mathbf{\varepsilon} \overline{\mathbf{E}}^{T}\right) \\
& =\boldsymbol{\Phi} \mathbf{Q} \overline{\mathbf{Q}}^{T} \bar{\Phi}^{T}+\boldsymbol{\varepsilon} \boldsymbol{\Phi} \mathbf{Q} \overline{\mathbf{E}}^{T}+\mathbf{\varepsilon} \mathbf{E} \overline{\mathbf{Q}}^{T} \bar{\Phi}^{T}+\boldsymbol{\varepsilon}^{2} \mathbf{E} \overline{\mathbf{E}}^{T},
\end{aligned}
$$

the latter term of which is very small and negligible. As such, $N \mathbf{R}=\Phi \mathbf{Q} \overline{\mathbf{Q}}^{T} \bar{\Phi}^{T}+O(\varepsilon)$, and if eigenvalues are distinct, the eigenvectors of $\mathbf{R}$ are perturbed by $O(\varepsilon)$, such that $\mathbf{v}_{i}=\phi_{i}+\varepsilon \mathbf{e}_{i}$.

Suppose, then, with small noise correction, the $\operatorname{COM}$ is $\mathbf{v}_{i}=$ $\underline{\phi}_{i}+\varepsilon_{i}$. Then $\mathbf{z}(t)=\mathbf{V} \mathbf{p}(t)=\left[\underline{\phi}_{1}+\varepsilon \mathbf{e}_{1}, \underline{\phi}_{2}+\varepsilon_{2}, \cdots, \underline{\phi}_{M}+\varepsilon \mathbf{e}_{M}\right] \mathbf{p}$, where $\mathbf{p}(t)$ and $\mathbf{V}$ are the noise polluted complex-orthogonal modal coordinate vector and modal matrix as obtained by COD. By orthogonality of the COMs, $\mathbf{p}(t)=\overline{\mathbf{V}}^{T} \mathbf{z}(t)$, whence

$$
p_{i}(t)=\underline{\phi}_{i}^{T} \mathbf{z}(t)+\varepsilon \overline{\mathbf{e}}_{i}^{T} \mathbf{z}(t)=q_{i}(t)+\varepsilon \overline{\mathbf{e}}_{i}^{T} \mathbf{z}(t)
$$

using $\underline{\phi}_{i}^{T} \underline{\phi}_{j}=\delta_{i j}$ (discrete normalized case). The error vector $\mathbf{e}_{i}$ can be written in terms of linear normal modes by using the expansion theorem [28]: $\mathbf{e}_{i}=\sum_{j=1, j \neq i}^{M} r_{j} \underline{\phi}_{j}$. Hence,

$$
p_{i}(t)=q_{i}(t)+\varepsilon \sum_{j=1, j \neq i}^{M} r_{j} q_{j}(t)
$$

Thus the noise contaminated complex orthogonal modal coordinate $p_{i}(t)$ is polluted by other normal modal coordinates depending on the deviation of the COM $\mathbf{v}_{i}$ from the true $\phi_{i}$, as well as the relative strengths of linear normal modal components $q_{j}(t)$. However, small-noise contaminated $p_{i}(t)$ and $\mathbf{v}_{i}$ should be representative of the "pure" $q_{i}(t)$ and $\phi_{i}$. If the "true" underlying modes $\underline{\phi}_{i}$ are not orthogonal, then an additional contribution of $q_{j}(t)$ to $p_{i}(t)$ appears in equation $(8)$.

\subsection{Traveling and Standing Waves}

A complex modal vector has a real and imaginary part. Generally, some standing occurs in the real modal motion if the real and imaginary parts of the complex mode have something in common, or if they are not the same in magnitude. To this end, the "traveling index" was defined [1] as the reciprocal of the condition number between the real and imaginary components of the complex mode. Pure traveling waves will have orthogonal components of the same magnitude, leading to a condition number of 1 , and hence a traveling index of one. (However, a traveling index of 1 may not necessarily imply a globally traveling wave.
A wave confined to travel, or "slosh", within a fixed range can also have a traveling index of 1.) Deviations in the magnitudes or directions of the complex vector parts will lead to larger condition numbers. Collinear vectors (completely dependent), or of greatly differing magnitudes, will have large condition numbers, and hence small traveling indices. As the traveling index approaches zero, there is essentially one independent vector, representing purely standing motion. Wave motions can be further dissected into traveling and standing parts [1].

\section{Nematode Studies}

Next we study the characteristics of nematode movements using COD. Presented here are results for an individual wild nematode, with an average length of $0.824 \mathrm{~mm}$, corresponding to "wild type worm 16" of the database which can be linked off of reference [25].

We monitored the worm posture using the Wormtracker system $[25,26]$ at Caltech, in which the C. elegans were videotaped in an optical tracking microscope. The videos are processed such that $(\hat{x}(t), \hat{y}(t))$ (planar) positions of 13 equally spaced virtual markers on the worms are identified, sampled and recorded. About four minutes of worm motion data are typically sampled at 0.1666 seconds per sample (1407 total time samples). This process can be done for wild and mutant nematodes. Post processing of these data using an Analyzer program (Matlab) [26] produces motion parameters such as velocities, bending frequencies, wavelengths, and track amplitudes (based on the size of the bounding box on a swimming worm). These quantitative characteristics can then be attached to the descriptions of various mutant behaviors [25], and compared to similar quantities obtained via the COD.

Let $\hat{\mathbf{x}}(t)$ and $\hat{\mathbf{y}}(t)$ be arrays of the $(\hat{x}(t), \hat{y}(t))$ coordinates of the 13 virtual markers on the wiggling worm. In preparation for the COD, first the center of mass of each sample of $\hat{\mathbf{x}}\left(t_{i}\right)$ and $\hat{\mathbf{y}}\left(t_{i}\right)$ data, for $i=1, \ldots, 1407$, was translated to the origin. For each sample configuration, the principal axes were found by applying POD [11] to the 13 marker positions in the $\hat{x}$ and $\hat{y}$ space. Then the worm was rotated so that its principal axis lined up with the horizontal axis, while keeping a record of each rotation angle $\boldsymbol{\theta}\left(t_{i}\right)$. This produced, for each time sample of each measured marker, a new $(x, y)$ coordinate, such that $x$ specifies the axial position and $y$ indicates the transverse position of a marker. (See Figure 1.)

The preliminary COD analysis was based on only the $y$ (transverse) data, to ease visualization. As such, column $i$ of the ensemble $Y$ was built from the $y$ values of the 13 markers at time $t_{i}$, with marker one corresponding to the head. The complex analytic ensemble $\mathbf{Z}$ was then created, and the COD was performed. The COVs indicated that two or three complex modes were above the noise level (Figure 2). The dominant COV suggests a dominant mean modal amplitude of about $0.16 \mathrm{~mm}$. For a 

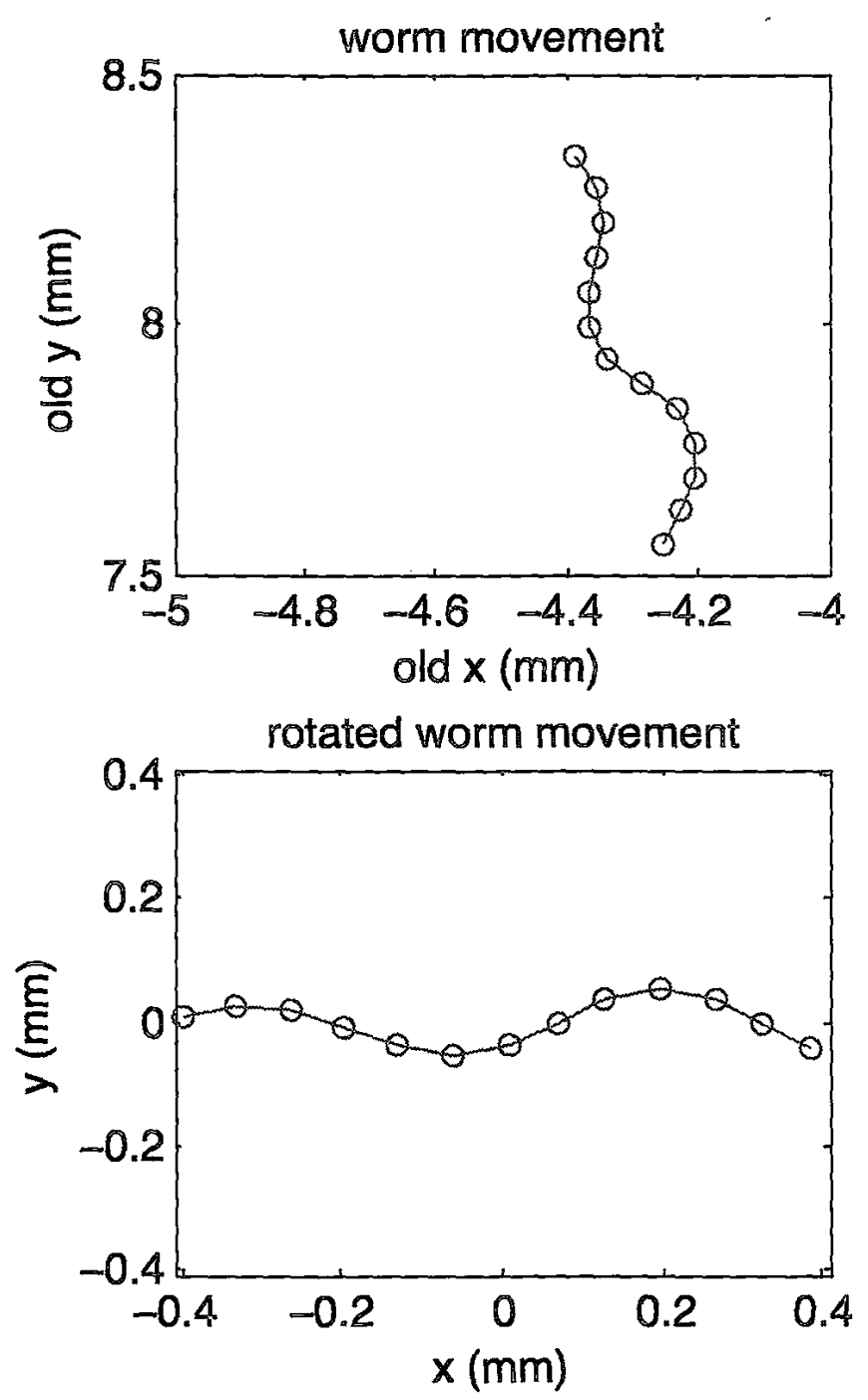

Figure 1. Above is the capiured worm, arbitrarily located and oriented, where "old $x$ " and "old $y$ " refer to $\hat{x} \hat{y}$. The head points down. The coordinates are translated to the origin and rotated, as shown below, where $y$ indicates the transverse deflection of the worm markers. The head points right.

worm of about $1 \mathrm{~mm}$ length, this is indicates a track peak to peak oscillation of about one third the worm length for the primary mode. The Analyzer program produced a mean track amplitude of about $14 \%$ from the aspect ratio of the instantaneous bounding box of the captured worm. The methods produce different measures of oscillation amplitude, and the results can be considered consistent. The mode shapes and their modal coordinate dynamics were examined. The first mode (Figure 3(top)), with an oscillatory modulation, represents motion that alternates between the solid and dashed lines, and their negatives, describing a traveling wave to the right. The head is to the left. The "trav-

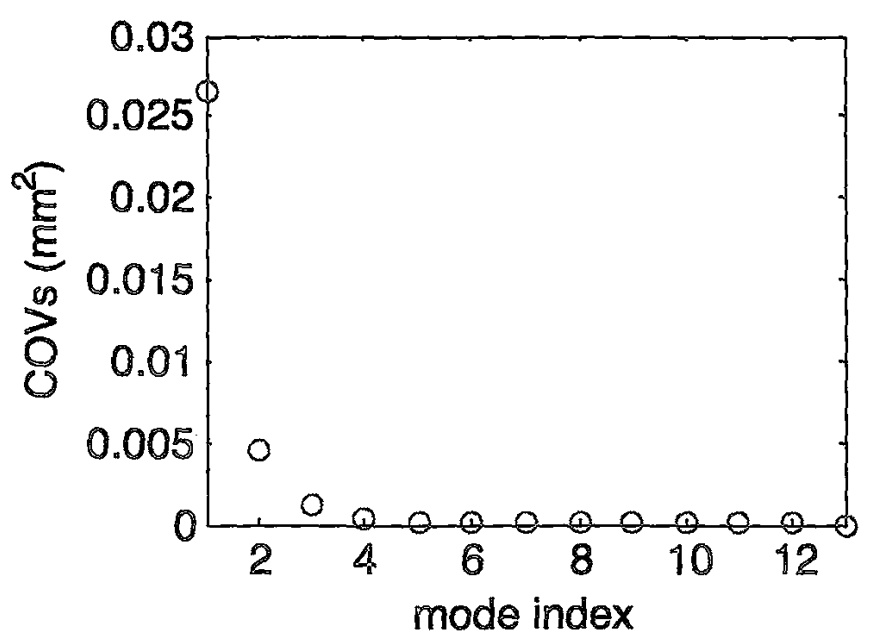

Figure 2. The COVs showing relative mean squared modal amplitudes.

eling index" of this mode is 0.9387 . From the complex plot of mode one (Figure 3(bottom)), we can estimate the wavelength as the portion of the worm's length that completes one full circle in the plot. Here the wavelength is about about 0.69 worm lengths.

It is informative to examine the modal coordinates, as well. The primary modal coordinate is persistently active; the mode represents locomotion. This modal coordinate has a consistent whirl in the complex plane. The real part of the modal coordinate is oscillatory, the frequency of which is the whirl rate of the complex coordinate. The frequency can be estimated from the mean complex whirl rate of the modal coordinate $\left(f_{w}=0.31\right.$ $\mathrm{Hz}$, with a standard deviation of 0.11$)$ or the FFT $\left(f_{F F T}=0.32\right.$ $\mathrm{Hz}$ ) of the real part of the complex coordinate. The mean frequency estimated by the Analyzer program (short time FFT of nematode bending) is $0.31 \mathrm{~Hz}$. The wave speed is estimated as $v_{w}=\lambda f_{w}=0.21$ worm lengths per second, or $0.173 \mathrm{~mm} / \mathrm{sec}$ on average. The instantaneous whirl rate is computed and shown in Figure 4, indicating that the worm has considerable variation, with apparent cruising speeds, as well as short bursts and lulls. The spectrum is also shown in Figure 4, with the variation in oscillation rate contributing to the broadened frequency peak.

The second mode is shown in Figure 5. The traveling index is 0.3589 , and thus it has more standing qualities than the first mode. The second mode is intermittently active. The occurrence of activity in this mode is strongly correlated with changes in $\theta(t)$, suggesting that this mode represents a steering effort. This can be seen by looking at the heading angle and its change (red and blue curves of Figure 6, top), along with the real part of the second modal coordinate $q_{2}(t)$ (magenta curve, overlapping the blue curve). The time derivative of the heading angle is approximated over a 60-sample range, or about a 10 second range, which covers on average about three primary-mode cycles, and is scaled by a factor of 10 in Figure 6 for visualization. The real part of the 

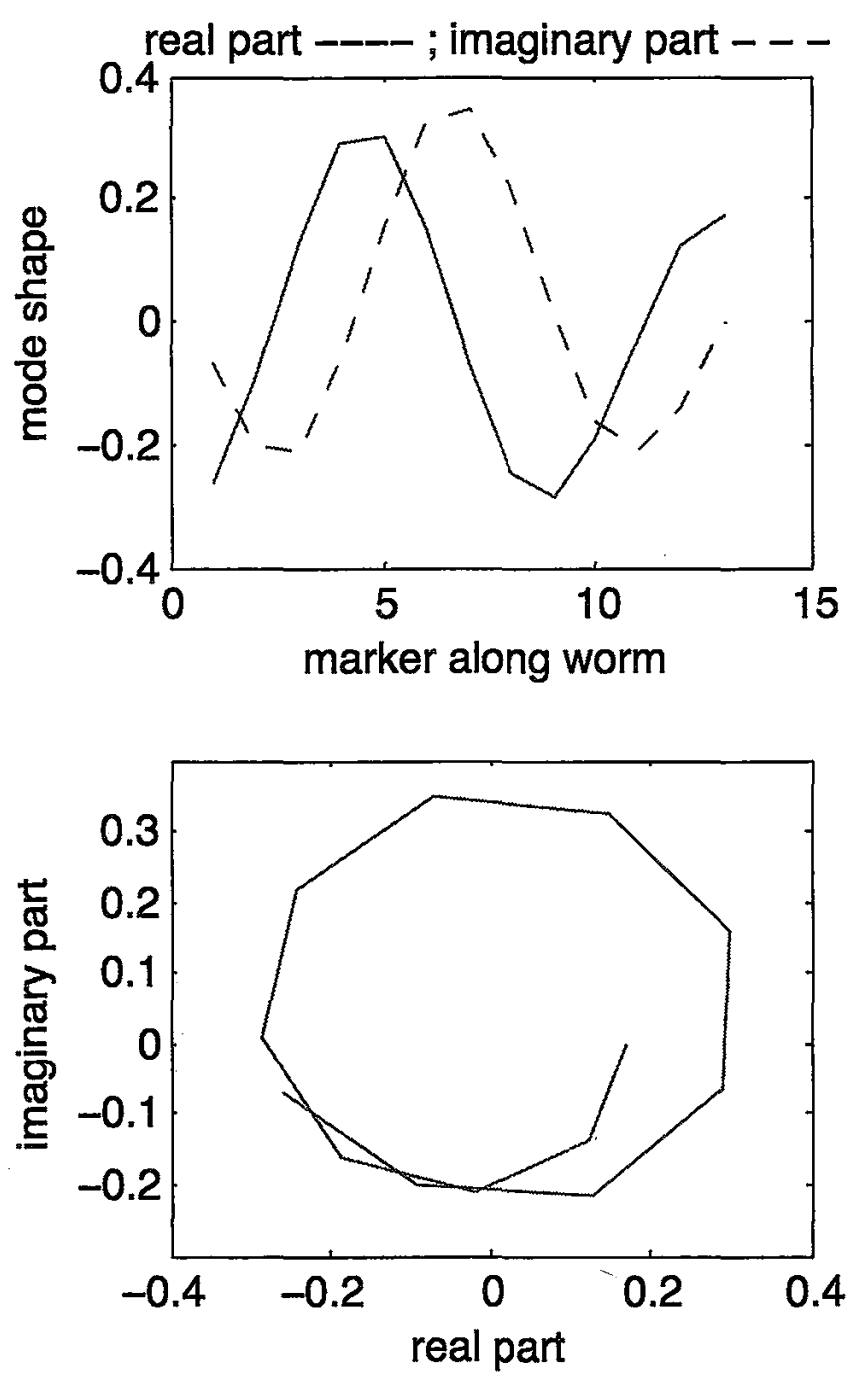

Figure 3. Top: (dominant) mode one, with real (solid line) and imaginary (dashed line) parts. (The vertical axes are magnified compared to Figure 1.) The head is at marker 1 and points left. Bottom: mode one in the complex plane, where the head is the end near coordinates $(-2,-1)$.

second modal coordinate is plotted as its moving average over 60 samples (or ten seconds). As the modal vectors are normalized to an amplitude of one, and are unitless, the modal coordinates are in $\mathrm{mm}$. The plot of the moving-averaged second modal coordinate $q_{2}(t)$ is scaled by a factor of ten to visually bring forth the correlation between $q_{2}(t)$ and the change in heading angle. The product $q_{2}(t) \dot{\theta}(t)$ between the moving-averaged second modal coordinateand the change in heading angle is shown in Figure 6 (bottom). The positive bias in this graph, and the correlation coefficient $\rho_{q_{2} \dot{\theta}}=E\left[\left(q_{2}(t)-\bar{q}_{2}\right)(\dot{\theta}(t)-\tilde{\dot{\theta}})\right] /\left(\sigma_{q_{2}} \sigma_{\dot{\theta}}\right)=0.70$, suggest strong correlation $(\rho=0$ indicates no correlation, and
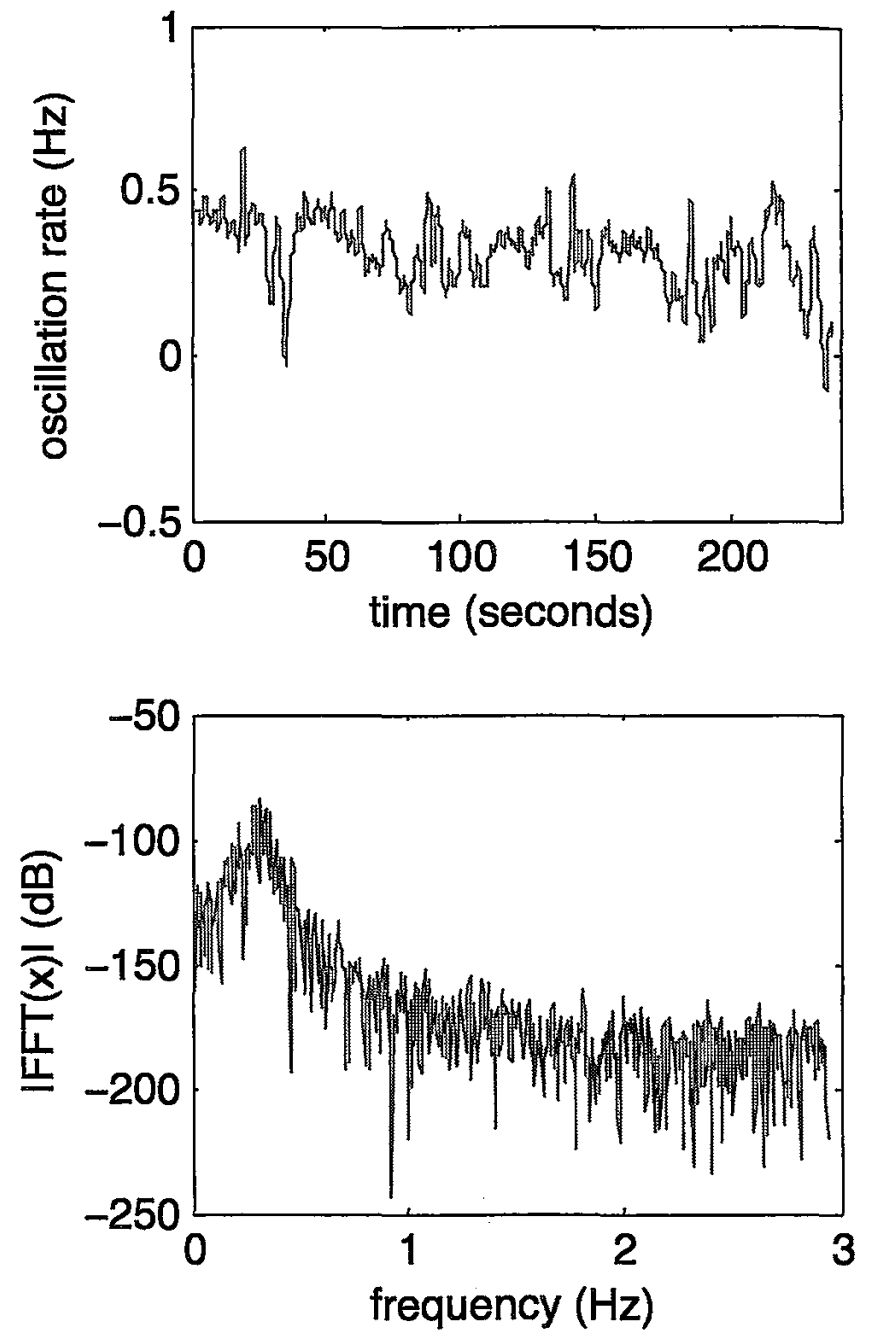

Figure 4. Top: dominant mode oscillation frequency, taken from the complex whirl rate, over time. Bottom: spectrum of the dominant modal coordinate, where $\mathrm{dB}$ is computed $20 \log q_{1}(t)$, and where $q_{1}$ is in $\mathrm{mm}$.

$|\rho|=1$ indicates perfect correlation [30]).

Indeed, the second-mode motion can be re-animated by modulating the second complex mode with the second complex modal coordinate, such that the activity of the second mode is isolated, and by adding $\theta(t)$ back into the motion, the correlation of second-mode activity and angular changes can be witnessed.

The third mode (not shown) is similar to the first, but the real and imaginary parts are oppositely phased, implying that it may be for reverse motion. At its low energy level, its modal coordinate is too noise polluted to verify this. 


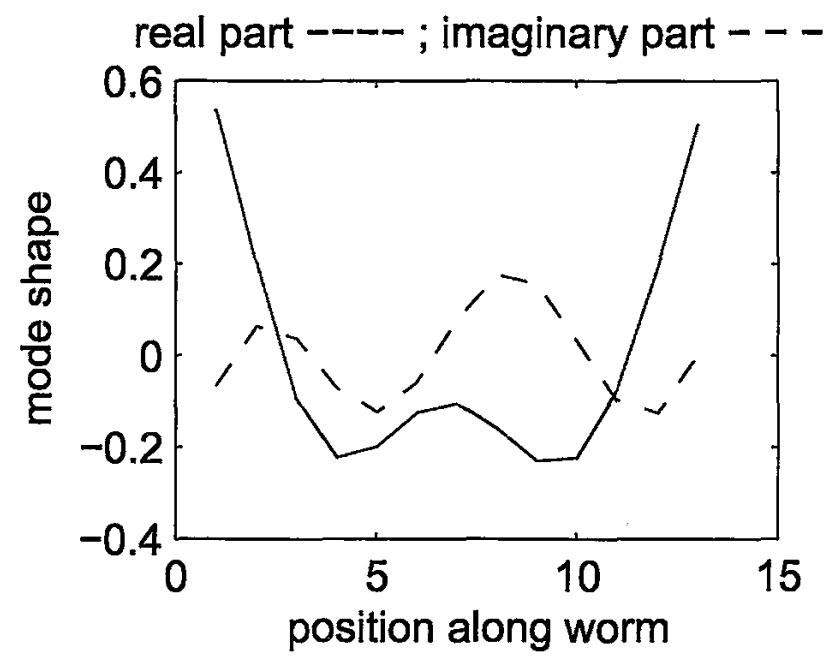

Figure 5. Mode two, with real (solid line) and imaginary (dashed line) parts. (The vertical axes are magnified compared to Figure 1.)

\section{Conclusion}

In summary, the COD provides a means of obtaining wave parameters, such as a description of the wave shape, the wave length and associated frequency and wave speed. We have sketched the process of obtaining this information though the extraction of complex modes, and also the use of complex modal coordinates, and have made a brief assessment on how small levels of noise affect the decomposition.

In this work we demonstrated the the application of COD to undulatory bio-locomotion, in particular to the posturing of a wild $C$. elegans nematode. The COD indicates that the worm has a multi-modal posturing behavior, involving at least a locomotion mode and a steering mode. The locomotion mode is dominant in terms of its mean squared modal amplitude, and it is closer to a pure traveling waveform than the steering, secondary mode. The complex mode representations allow the worm's waveforms to be described with the two still real and imaginary parts, which is an alternative to animated descriptions of the waves. The characteristic wavelength of the primary mode was estimated in the complex plane. Frequency was obtained from the complex modal coordinate's complex whirl rate, and from its FFT. The results were consistent with results from the Caltech Analyzer program.

In future work, the COD will be used for quantifying normal and abnormal motions, which can then be connected to the study of genetic mutations on phenotypes associated with locomotion.
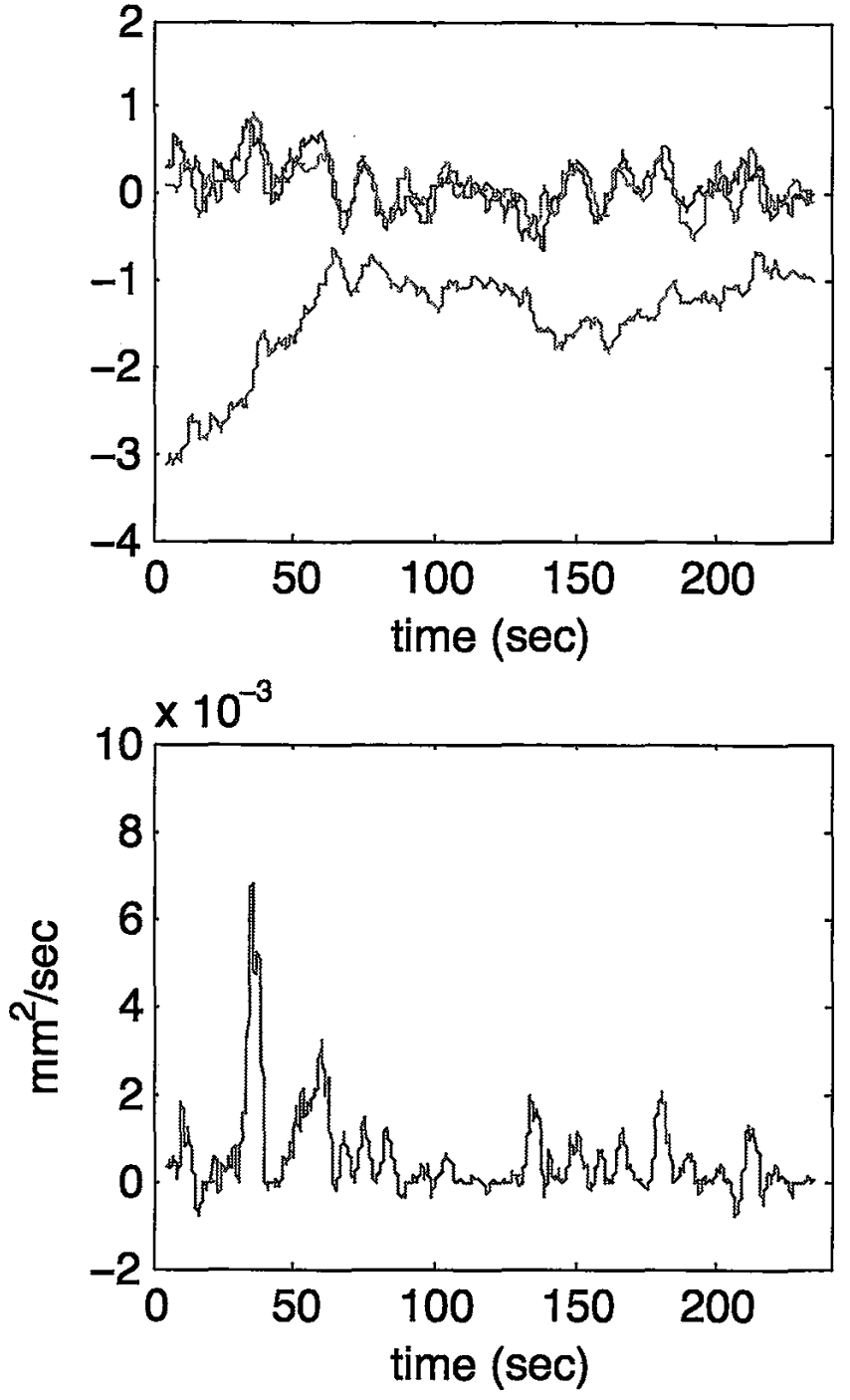

Figure 6. Top: the heading angle $\theta(t)$ (lower curve, in red) in radians; the scaled change in angle $10 \dot{\theta}(t)$ (blue, overlapping), in radians, computed by finite differences; and the real part of the scaled movingaveraged second modal coordinate $10 q_{2}(t)$ (magenta, overlapping) in $\mathrm{mm}$. Bottom: the product of the second modal coordinate and the change in heading angle. $q_{2}(t) \dot{\theta}(t)$, in $\mathrm{mm}^{2}$ per second.

\section{REFERENCES}

[1] B. F. Feeny, 2008, "A complex orthogonal decomposition for wave motion analysis", Journal of Sound and Vibration 310 (1-2) 77-90.

[2] Lumley, J. L., 1970, Stochastic Tools in Turbulence, Academic Press, New York.

[3] Berkooz, G., Holmes, P., and Lumley, J. L., 1993, "The proper orthogonal decomposition in the analysis of turbulent flows," Annual Review of Fluid Mechanics, Vol. 25, 
pp. 539-575.

[4] Cusumano, J. P., and Bai, B.-Y., 1993, "Period-infinity periodic motions, chaos, and spatial coherence in a 10 degree of freedom impact oscillator," Chaos, Solitons, and Fractals, Vol. 3, No. 5, pp. 515-535.

[5] Epureanu, B. I., Tang, L. S., and Paidoussis, M. P., 2004, "Exploiting chaotic dynamics for detecting parametric variations in aeroselastic systems," AIAA Journal 42 (4) 728735.

[6] FitzSimons, P., and Rui, C., 1993, "Determining low dimensional models of distributed systems," Advances in Robust and Nonlinear Control Systems, ASME DSC-Vol. 53, pp. 9-15.

[7] Kerschen, G., Golinval, J. C., Vakakis, A. F., and Bergman, L. A., 2005, "The method of proper orthogonal decomposition for dynamical characterization and order reduction of mechanical systems: An overview," Nonlinear Dynamics 41(1-3) 147-169.

[8] Yasuda, K., and Kamiya, K., 1997, "Experimental identification technique of nonlinear beams in time domain," ASME Design Engineering Technical Conferences," Sacramento, on CD-ROM.

[9] Ma, X., Azeez, M. A. F., and Vakakis, A. F., 2000, "Nonlinear normal modes and nonparametric system identification of nonlinear oscillators," Mechanical Systems and Signal Processing 14 (1) 37-48.

[10] Liang, Y. C. Lee, H. P., Lim, S. P., Lin, W. Z., Lee, K. H., and Wu, C. g., 2002, "Proper orthogonal decomposition and its applications-part 1: theory," Journal of Sound and Vibration 252 (3) 527-544.

[11] Feeny, B. F., and Kappagantu, R., 1998, "On the physical interpretation of proper orthogonal modes in vibrations," Journal of Sound and Vibration 211 (4) 607-616.

[12] Feeny, B. F., 2002, "On the proper orthogonal modes and normal modes of continuous vibration systems," Journal of Vibration and Acoustics 124(1) 157-160.

[13] Kerschen G., Golinval J. C., 2002, "Physical interpretation of the proper orthogonal modes using the singular value decomposition," Journal of Sound and Vibration 249(5) 849. 865.

[14] Feeny, B. F., and Liang, Y., 2003, "Interpreting proper orthogonal modes in randomly excited vibration systems," Journal of Sound and Vibration 265(5) 953-966.

[15] NIH-National Human Genome Research Institute. "International genome team deciphers genetic instructions for a complete animal," ScienceDaily, 11 December 1998. Retrieved Feb. 6, 2008, from http://www.sciencedaily.com/ releases/1998/12/981211084627.htm

[16] Mendel, J. E., Korswagen, H. C., Liu, K. S., Hajdu-Cronin, Y. M., Simon, M. I., Plasterk, R. H. A., and Sternberg, P. W., 1995, "Participation of the protein Go in multiple aspects of behavior in C. elegans," Science 267: 1652-1655.
[17] Jorgensen E. M. GABA. WormBook. 2005 Aug 31:1-13. http://www.wormbook.org

[18] Bastiani C., Mendel J., 2006, "Heterotrimeric G proteins in C. elegans," WormBook. 2006 Oct 13:1-25. http://www.wormbook.org

[19] Rand, J. B., "Acetylcholine," WormBook. 2007 Jan 30:1-21. http://www.wormbook.org

[20] Chase D. L. and Koelle M. R. 2007, "Biogenic amine neurotransmitters in C. elegans," WormBook. 2007 Feb 20:115. http://www.wormbook.org

[21] Li, W., Feng, Z., Sternberg, P. W., and Xu, X. Z. S., 2006, "A C. elegans stretch-sensitive neuron revealed by a mechanosensitive TRP channel homologue," Nature 440: 684-687.

[22] Van Buskirk, C. and Sternberg, P. W., 2007, "EGF signaling induces behavioral quiescence in $C$. elegans," Nature Neuroscience 10:1300-1307.

[23] Karbowski, J., Cronin, C. J., Seah, A., Mendel, J. E., Cleary, D. and Sternberg, P. W. 2006, "Conservation rules, their breakdown, and optimality in Caenorhabditis sinusoidal locomotion," J. Theoretical Biology 242:652-669.

[24] Karbowski, J., Schindelman, G., Cronin, C. J., Seah, A., and Sternberg, P. W. 2007, "Systems level circuit model of $C$. elegans undulatory locomotion: mathematical modeling and molecular genetics," $J$. Computational Neurosci., in press. doi 10.1007/s10827-007-0054-6

[25] Cronin, C. J., Mendel, J. E., Muhktar, S., Kim, Y.-M., Stirbl, R. C., Bruck, J., and Sternberg, P. W. 2005, "An automated system for measuring parameters of nematode sinusoidal movement," BMC-Genetics 6: 5 .

[26] Z. Feng, C. J. Cronin, J. H. Wittig, Jr., P. W. Sternberg, and W. R. Schafer, 2004, "An imaging system for standardized quantitative analysis of C. elegans behavior," BMC Bioinformatics 5115.

[27] Oppenheim, A. V., and Schafer, R. W., 1989, Discrete-Time Signal Processing, Prentice Hall, Englewood Cliffs, NJ.

[28] Meirovitch, L., 1997, Principles and Techniques in Vibrations Prentice Hall.

[29] Ginsberg, J., 2001, Mechanical and Structural Vibrations, Wiley, New York.

[30] Newland, D. E., 1993, An Introduction to Random Vibrations, Spectral and Wavelet Analysis, third edition, Longman Scientific and Technical, Singapore. 